\title{
High-resolution 3T magnetic resonance imaging and histological analysis of capsuloligamentous complex of the first metatarsophalangeal joint
}

\author{
Jin-e Wang ${ }^{1}$, Rong-jie Bai ${ }^{1,2^{*}}$, Hui-li Zhan ${ }^{2}$, Wen-ting Li ${ }^{3}$, Zhan-hua Qian², Nai-li Wang ${ }^{3}$ and Yuming Yin ${ }^{4}$
}

\begin{abstract}
Background: There are discrepancies in the understanding of the structure of the capsuloligamentous complex of the first metatarsophalangeal joint (MTPJ); this study aims to investigate the differences with previous anatomical reports of high-resolution $3 \mathrm{~T}$ magnetic resonance imaging (MRI) and histological analysis in illustrating the structure of the capsuloligamentous complex of the first MTPJ.
\end{abstract}

Methods: Nine fresh frozen cadaveric feet specimens (from two women and three men; aged 32 to 58 years) were used in this study. All specimens underwent MR examination with T1-weighted imaging and T2-weighted spectral attenuated inversion recovery in three planes. Subsequently, all cadaveric feet specimens were sliced into 2-mm-thick sections. The MRI features of the capsuloligamentous complex of the first MTPJ were analyzed in these specimens. Hematoxylin-eosin and Masson's trichrome staining methods were used to explore the histologic features of the capsuloligamentous complex of the first MTPJ.

Results: Different from most previous studies, our results showed that the plantar plate could be divided into four portions including the central portion of the plantar plate, the intersesamoid, the sesamoid phalangeal and the metatarsosesamoid ligaments. The normal central portion of the plantar plate could be clearly visualized in the sagittal and coronal plane MR images. The intersesamoid ligament is a continuation of the central portion of the plantar plate on the sagittal plane on the gross specimen, the MR imaging, and the histological examination. On the coronal plane of the gross specimen and MR imaging, the sesamoid phalangeal ligaments and the central portion of the plantar plate can be seen as separate ligaments, but they appeared interwoven with the same continuous collagenous fibers on the histological analysis.

Conclusion: High-resolution 3T MRI allows accurate demonstration of the different anatomical details of the capsuloligamentous complex of the first MTPJ from previous anatomical reports. The histological analysis provides further understanding of the structures of the capsuloligamentous complex of the first MTPJ from previous studies.

Keywords: Anatomy, Magnetic resonance imaging, Histological analysis, Capsuloligamentous complex, First metatarsophalangeal joint, Foot

*Correspondence: bairongjie@126.com

1 Department of Radiology, Peking University Fourth School of Clinical Medicine, Beijing 100035, China

Full list of author information is available at the end of the article

\section{Background}

The capsuloligamentous complex of the first MTPJ is composed of joint capsule, multiple ligaments, plantar plate, and supporting structures [1-3]. It is the main original author(s) and the source, provide a link to the Creative Commons licence, and indicate if changes were made. The images or other third party material in this article are included in the article's Creative Commons licence, unless indicated otherwise in a credit line to the material. If material is not included in the article's Creative Commons licence and your intended use is not permitted by statutory regulation or exceeds the permitted use, you will need to obtain permission directly from the copyright holder. To view a copy of this licence, visit http://creativecommons.org/licenses/by/4.0/. The Creative Commons Public Domain Dedication waiver (http://creativeco mmons.org/publicdomain/zero/1.0/) applies to the data made available in this article, unless otherwise stated in a credit line to the data. 
stabilizer of the first MTPJ, and injuries of the capsuloligamentous complex of the first MTPJ may lead to "turf toe" [4]. However, there are discrepancies in the understanding of the structure of the capsuloligamentous complex of the first MTPJ, which make the accurate diagnosis of turf toe a challenge. Unfamiliar with the complexity of the normal anatomy and anatomic variation may lead to failure in recognizing the injury. If a delayed diagnosis and treatment occur, subsequent prolonged recovery may result in the instability of the joint and may lead to significant functional disability [5-9].

Previously, magnetic resonance imaging (MRI) has been shown to be valuable for the visualization of the fine anatomic structures of the capsuloligamentous complex of the first MTPJ and evaluation of tendons, ligaments, and cartilaginous injuries related to turf toe $[2,10-15]$. In this study, using human fresh frozen cadaveric feet specimens and correlating with the high-resolution 3T MR images and histological evaluation in the visualization and analysis of the anatomy of the capsuloligamentous complex of the first MTPJ, we found that the components of the plantar plate, the relations between the intersesamoid ligament and the central portion of the plantar plate and the relations between the sesamoid phalangeal ligaments and the central portion of the plantar plate were different with previous reports.

\section{Methods}

The study was conducted in accordance with the Declaration of Helsinki and was approved by the Ethics Committee of the Institutional Review Board of Beijing Jishuitan Hospital (201703-10). The authors certify that they have obtained all appropriate consent forms, which were signed by the family members of the donor-cadavers.

\section{Cadaveric feet specimens}

Nine fresh frozen cadaveric feet specimens were obtained from five cadavers (two women and three men; aged from 32 to 58 years at death, mean age 42 years) provided by the Institute of Basic Sciences, Chinese Academy of Medical Sciences. All specimens included the whole ankle joint and foot and were kept at $-42{ }^{\circ} \mathrm{C}$ (Haier BioMedical, DW-40W100, Qingdao, China). All specimens were allowed to thaw for $24 \mathrm{~h}$ at room temperature before the MRI analysis. A anteroposterior and lateral X-ray analysis of nine feet specimens was performed to exclude the presence of articular disorders or osseous abnormalities.

\section{Magnetic resonance imaging}

MR was performed with a 3T MRI Unit (5680 DA Best, Philips Medical Systems, Netherlands). A 16-channel head-and-neck receiver-only coil was used, centered on the forefoot by the laser mark. The MRI examination includes the following sequences: T1-weighted image (T1WI) and T2-weighted spectral attenuated inversion recovery (T2-SPAIR) in the coronal, transverse, and sagittal planes. In order to evaluate the detail of the capsuloligamentous complex of the first MTPJ, two specimens had additional sequences with smaller field of view (FOV: $6 \mathrm{~cm} \times 6 \mathrm{~cm}$ ) including T1WI and proton densityweighted imaging with fat suppression (PD-FS) in sagittal and coronal planes. Detailed parameters are listed in Additional file 1: Table S1.

\section{Anatomic specimen preparation}

All cadaveric feet were frozen for at least $24 \mathrm{~h}$ at $-42{ }^{\circ} \mathrm{C}$ after obtaining the MR imaging. Subsequently, specimens were sliced into 2-mm-thick sections with a stainlesssteel band saw (American Meat Equipment Corp, Montebello, USA); the section thickness is the same as the MR images. Nine specimens were sectioned as follows: sagittal $(n=3)$, transverse $(n=3)$, and coronal $(n=3)$ planes. Photographs of each section of the specimens were obtained.

\section{Histological examination}

The histologic slice was fixed in $10 \%$ neutral phosphatebuffered formalin (NBF), dehydrated in ascending ethanol, decalcified with ethylenediaminetetraacetic acid (EDTA) and formic acid, and embedded into a paraffin wax block for histological examination. The $4-\mu \mathrm{m}$ sequential transverse sections were stained with hematoxylin and eosin (HE) and Masson's trichrome at original magnifications of $4 \times$ and $200 \times$ and then observed under the light microscope.

\section{Magnetic resonance imaging and light microscopic observation: anatomic comparison and analysis}

All the MR images were interpreted by two musculoskeletal radiologists with 10-15 years of experience. The features of the capsuloligamentous complex of the first MTPJ on MRI were compared with those derived from examination of the anatomic segments of the cadaveric specimens.

Identification of each structure of the capsuloligamentous complex of the first MTPJ on MRI was by confirming the linear or curved hypointense signal structure in the expected location on MR imaging. Diagrams were drawn according to the appearance of the corresponding MR images.

Two musculoskeletal radiologists measured the capsuloligamentous complex of the first MTPJ of the MR images independently. All measurements were taken three times in the middle of their respective courses of the optimal MRI images plane $[1,16]$. 
The data are presented as mean \pm standard deviation. Interobserver agreement regarding measurements was assessed using the intraclass correlation coefficient (ICC). ICC value less than 0.20 was rated as slight agreement, between 0.21 and 0.40 as fair agreement, between 0.41 and 0.60 as moderate agreement, between 0.61 and 0.80 as good agreement, and between 0.81 and 1.00 as very good interobserver agreement.

The structure of the capsuloligamentous complex of the first MTPJ was identified by confirming the collagen fibers on histological examination including the central portion of the plantar plate, the intersesamoid, the sesamoid phalangeal, the accessory sesamoid ligaments, and abductor and adductor hallucis tendons.

\section{Results}

\section{Anatomy of the capsuloligamentous complex of the first} MTPJ

Analysis of the gross specimens confirmed that capsuloligamentous complex of the first MTPJ was composed of plantar plate, collateral ligaments, and supporting structures (Figs. 1-8).

\section{The plantar plate of the capsuloligamentous complex of the first MTPJ}

The plantar plate could be divided into four portions (central portion of the plantar plate, intersesamoid, sesamoid phalangeal and metatarsosesamoid ligaments) by the embedded sesamoids (Fig. 1).

The central portion of the plantar plate originated from the metatarsal neck and extended toward the plantar side of the proximal phalangeal base distally (Fig. 2). The posterior midline aspect of the plantar plate, locating between the medial and lateral sesamoids, was referred to as the intersesamoid ligament. At the periphery of the plantar plate, it was divided into proximal metatarsosesamoid ligament and distal sesamoid phalangeal ligament with the intervening sesamoids: the former one extended from the metatarsal neck and inserted on the proximal margin of sesamoid, the latter one originated from the

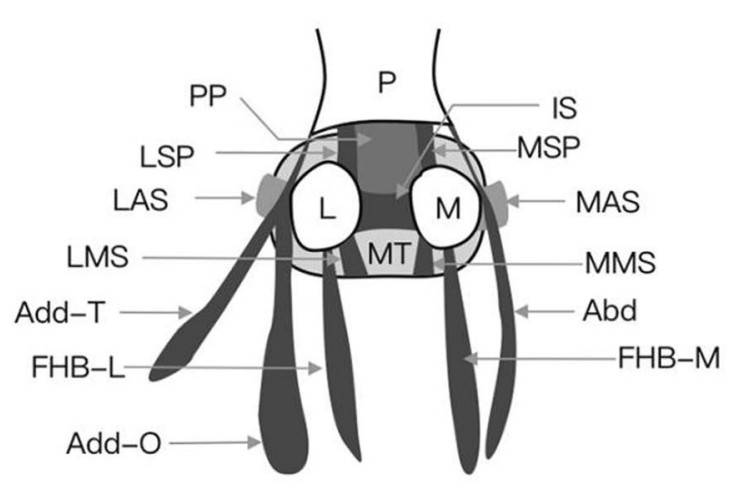

a

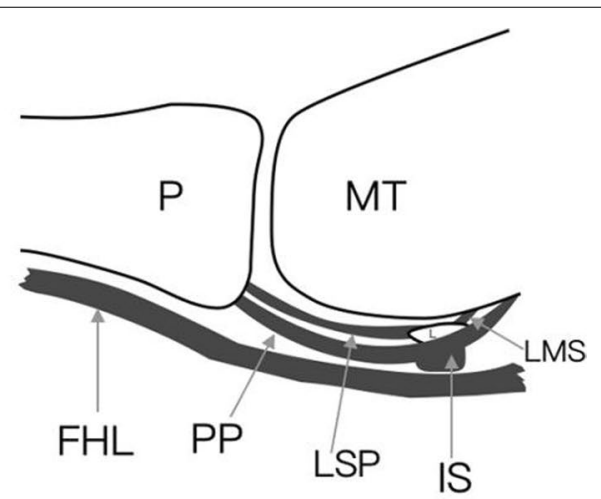

b

Fig. 1 Schematic diagram of capsuloligamentous complex of the first MTPJ in the foot. a Anatomy of the first MTPJ, dorsal view; $\mathbf{b}$ Anatomy of the first MTPJ, medial side of the proximal midsagittal images. (Abd=abductor hallucis tendon; Add-O=oblique head of adductor hallucis tendon; Add-T = transverse head of adductor hallucis tendon; FHB-M = medial head of flexor hallucis brevis tendon; FHB-L= lateral head of the flexor hallucis brevis tendon; $F H L=$ flexor hallucis longus tendon; IS= intersesamoid ligament; $L=$ lateral sesamoid; LAS= lateral accessory sesamoid ligament; LMS = lateral metatarsosesamoid ligament; LSP = lateral sesamoid phalangeal ligament; $M=$ medial sesamoid; MAS= medial accessory

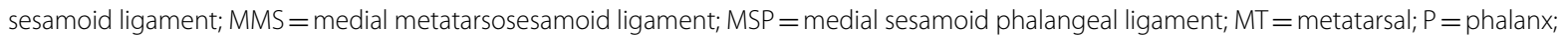
$\mathrm{PP}=$ central portion of the plantar plate)

(See figure on next page.)

Fig. 2 The central portion of the plantar plate and intersesamoid ligament of the first MTPJ from a left foot specimen (33 years old). a Midsagittal schematic diagram of first MTPJ; $\mathbf{b}$ sagittal anatomic slice; c sagittal T1WI image of the MTPJ; d sagittal PD-FS image of the MTPJ. White curved arrow: The central portion of the plantar plate coursed from metatarsal neck (white short arrow) to the proximal phalangeal base. Note a normal, smooth, well-defined distal recess (arrowhead) at the insertion of the proximal phalangeal base of the central portion of the plantar plate. (The white long arrow = flexor hallucis longus tendon; White triangle=The intersesamoid ligament). e and $\mathbf{f}$ HE and Masson's trichrome staining of the first MTPJ (original magnification $\times 4$ ). The white short arrow and arrowhead: the proximal and distal attachment of the central portion of the plantar plate. $\mathbf{g}$ and $\mathbf{h} \mathrm{HE}$ staining of the central portion of plantar plate and the intersesamoid ligament; They appeared interwoven with the same continuous collagenous fibers that with a small number of chondroid metaplasia (HE, original magnification $\times 200)$. (FHL $=$ flexor hallucis longus tendon; $\mathrm{IS}=$ intersesamoid ligament; $\mathrm{MT}=$ metatarsal; $\mathrm{P}=$ phalanx; $\mathrm{PP}=$ central portion of the plantar plate.) 


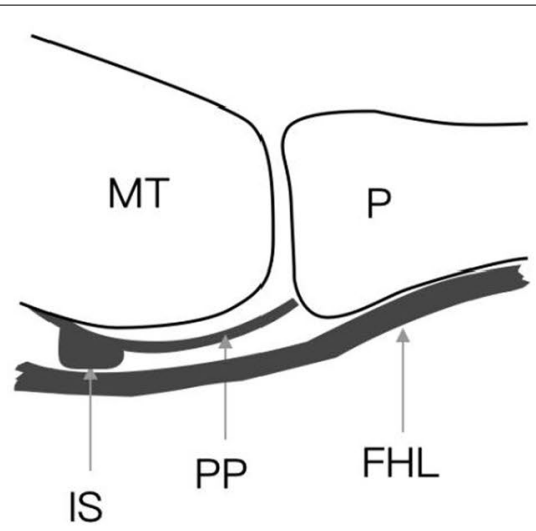

a

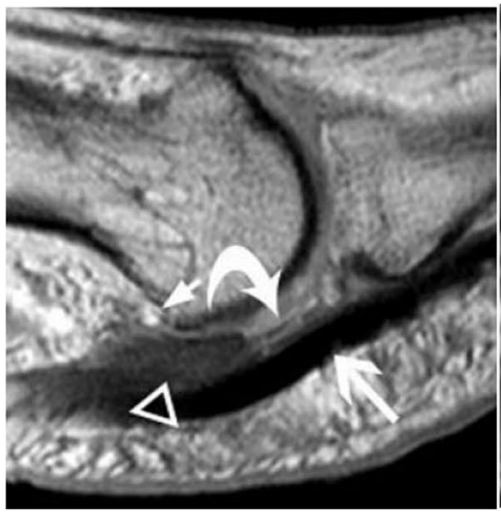

c

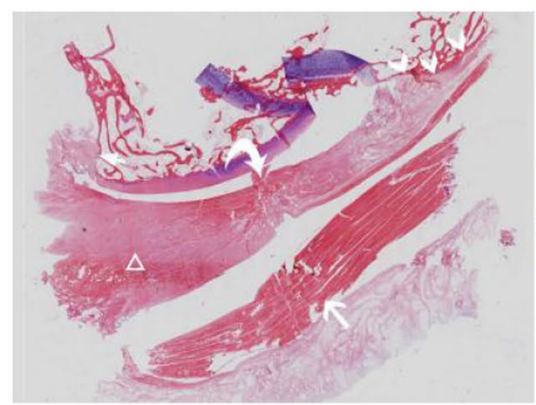

e

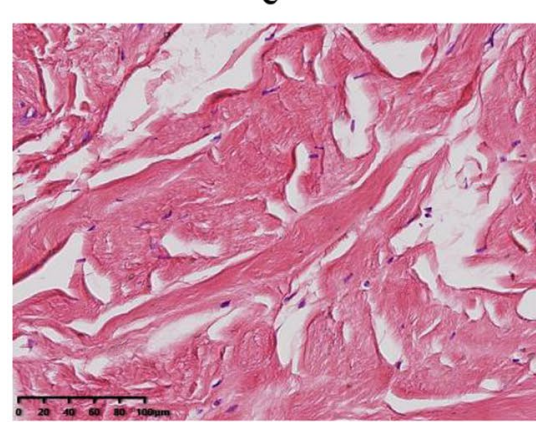

g

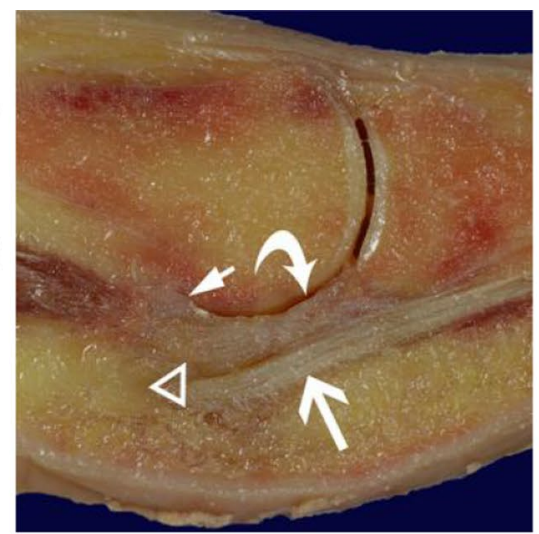

b

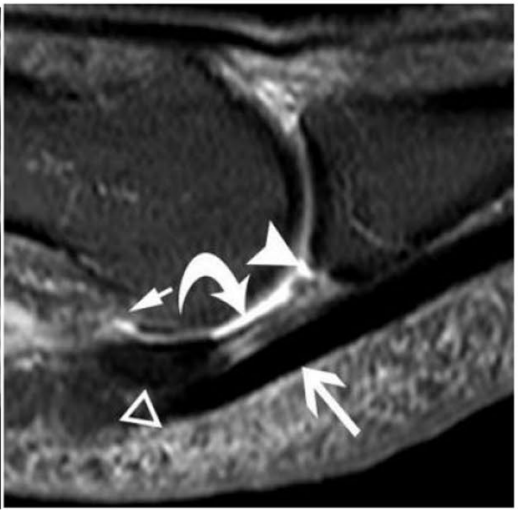

d

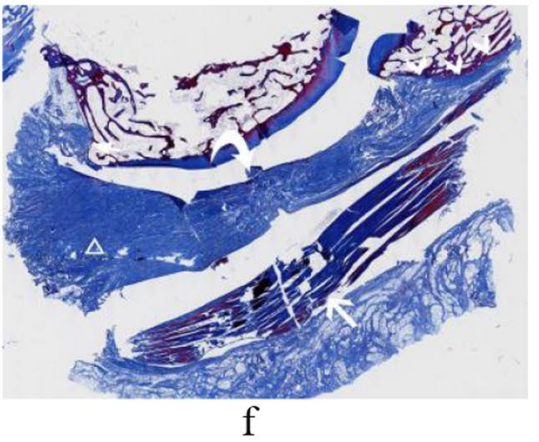

$\mathrm{f}$

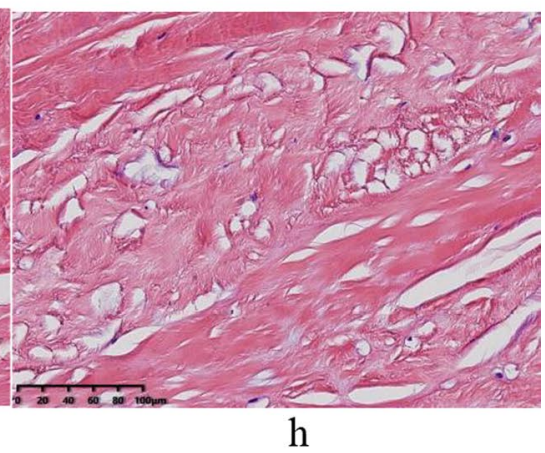

Fig. 2 (See legend on previous page.) 
distal aspect of the sesamoid to the proximal phalangeal base (Fig. 3). The central portion of the plantar plate was a separate fibrous band located between the medial and lateral sesamoid phalangeal ligaments in the coronal plane (Figs. 4 and 5), but it continued with the intersesamoid ligament in the sagittal plane (Fig. 2).

\section{The collateral ligaments of the capsuloligamentous complex of the first MTPJ}

The collateral ligaments consisted of two main collateral ligaments and two accessory sesamoid ligaments. The medial main collateral ligaments originated from the medial aspect of the metatarsal head to attach to the medial aspect of the proximal phalangeal base. The lateral main collateral ligaments originated from the lateral aspect of the metatarsal head to attach to the lateral aspect of the proximal phalangeal base (Fig. 6). The accessory sesamoid ligaments extended from the same proximal attachments with the main collateral ligaments to the periphery of the sesamoid bone (Fig. 7).

\section{The supporting structures of the capsuloligamentous complex of the first MTPJ}

The supporting structures of the capsuloligamentous complex of the first MTPJ were composed of the medial and lateral heads of the flexor hallucis brevis tendons and abductor and adductor hallucis tendons. The medial and lateral heads of the flexor hallucis brevis tendons attached to the plantar aspect of the medial and lateral sesamoids, respectively, and continued distally to attach to the proximal phalangeal base (Fig. 7). The abductor hallucis tendons attached to the medial side of the medial sesamoids, forming a conjoined tendon with the flexor hallucis brevis to insert into the plantar aspect of the medial side of the proximal phalangeal base. The adductor hallucis tendons attached to the lateral side of the lateral sesamoids, forming a conjoined tendon with the flexor hallucis brevis to insert into the plantar aspect of the lateral side of the proximal phalangeal base (Fig. 8).

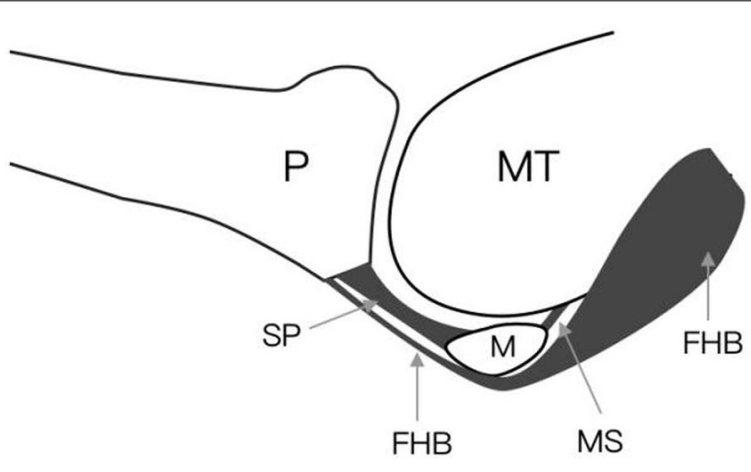

a

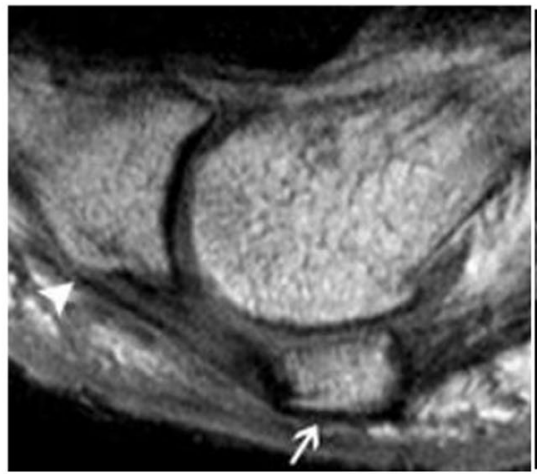

c

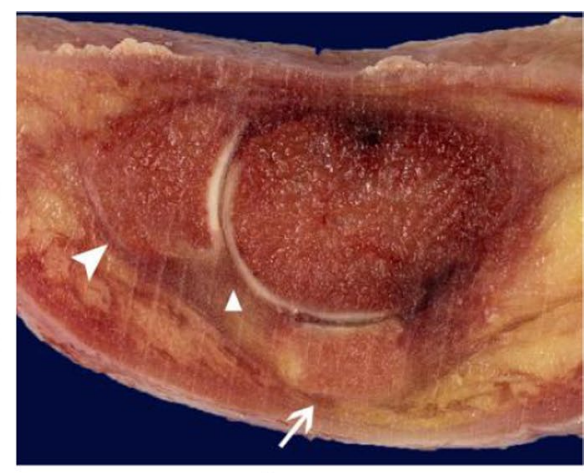

b

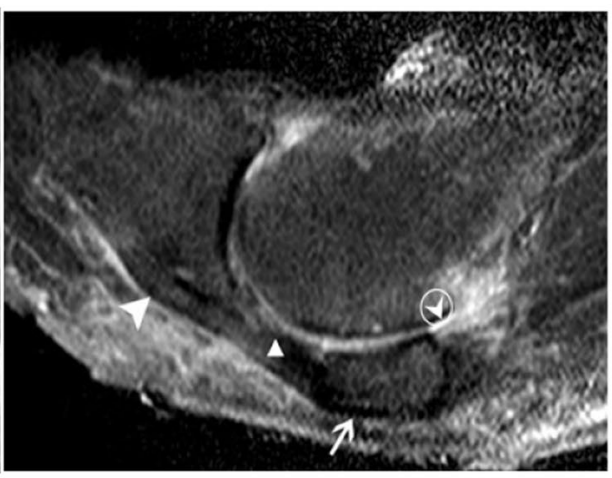

d

Fig. 3 The medial sesamoid phalangeal ligament, metatarsosesamoid ligament, the tendon of medial head of the flexor hallucis brevis from a left foot specimen (58 years old). a Parasagittal schematic diagram through the medial sesamoid of first MTPJ; $\mathbf{b}$ sagittal anatomic slice; $\mathbf{c}$ and $\mathbf{d}$ sagittal T1WI and PD-FS image of the MTPJ. White triangle=The medial sesamoid phalangeal ligament; white arrowhead in circle= The medial metatarsosesamoid ligament; white arrow: The tendon of medial head of the flexor hallucis brevis inserted to the plantar margin of the medial sesamoid, and continued distally to attach to the proximal phalangeal base (white arrowhead). (FHB=the flexor hallucis brevis; $\mathrm{M}=\mathrm{medial}$ sesamoid; $M S=$ metatarsosesamoid ligament; $M T=$ metatarsal; $P=$ phalanx; $S P=$ sesamoid phalangeal ligament) 


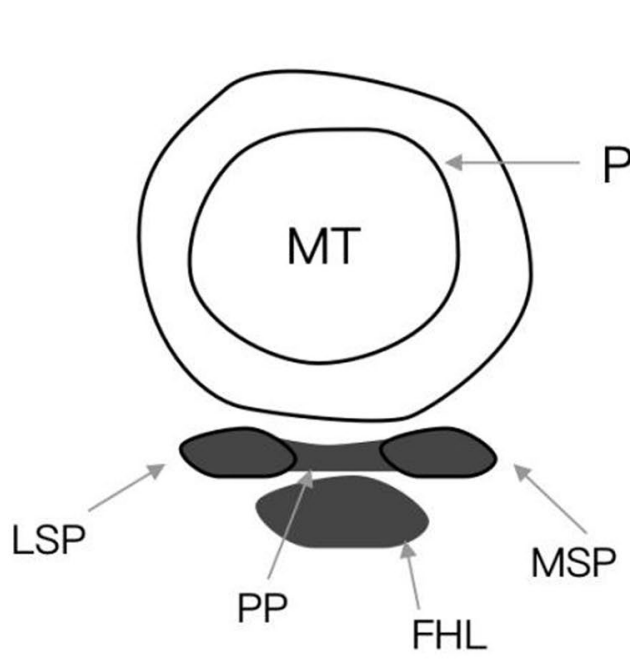

a

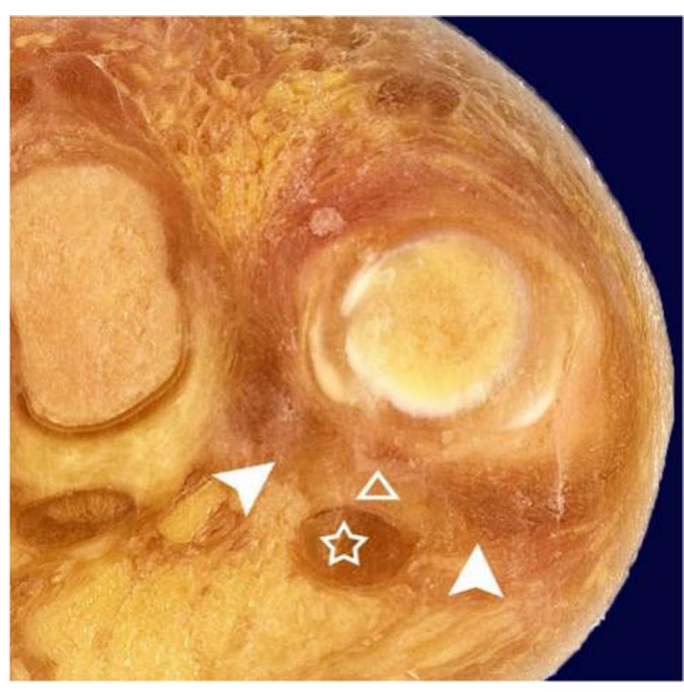

b

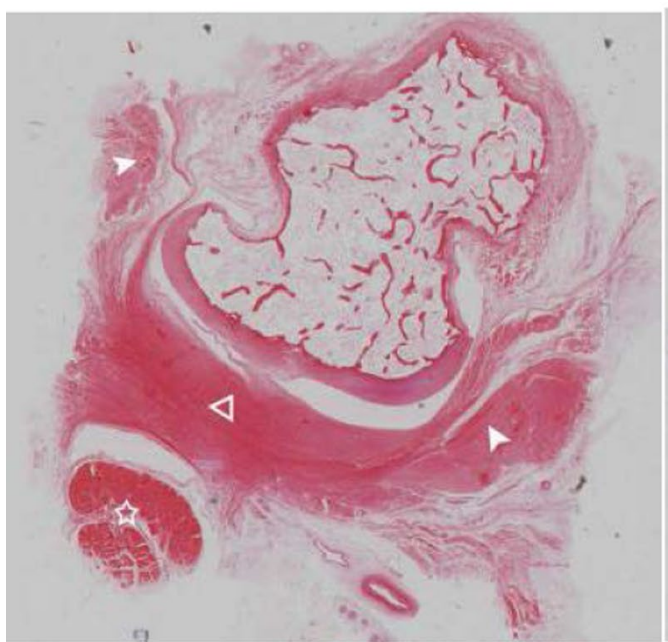

$\mathrm{c}$

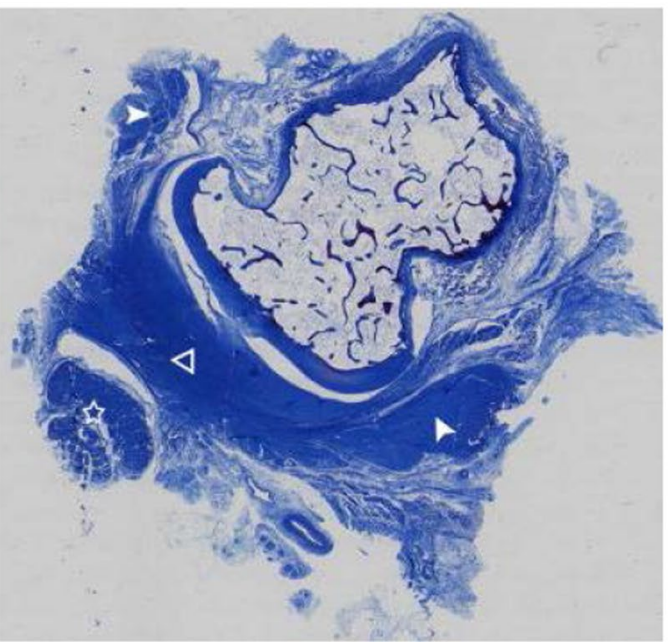

d

Fig. 4 The central portion of the plantar plate and sesamoid phalangeal ligaments of the first MTPJ from a right foot specimen ( 32 years old). a Coronal schematic diagram of first MTPJ; $\mathbf{b}$ coronal anatomic specimen; the central portion of the plantar plate (white triangle) was separate fibrous band located between the medial and lateral sesamoid phalangeal ligaments (white arrowheads); c and $\mathbf{d}$ HE and Masson's trichrome staining of the first MTPJ (original magnification $\times 4$ ). The central portion of the plantar plate (white triangle) and the sesamoid phalangeal ligaments (white arrowheads) were composed of the same continuous collagen fibers and showed eosinophilic features by HE, blue with Masson's trichrome staining, respectively. (White pentagram = the flexor hallucis longus tendon; FHL = flexor hallucis longus tendon; LSP = lateral sesamoid phalangeal ligament; $\mathrm{MSP}=$ medial sesamoid phalangeal ligament; $\mathrm{MT}=$ metatarsal; $\mathrm{P}=$ phalanx; $\mathrm{PP}=$ central portion of the plantar plate.)

\section{MRI investigation of the capsuloligamentous complex of the first MTPJ}

On MR images, the normal structures of the capsuloligamentous complex of the first MTPJ showed hypointense signal in all the sequences including T1-weighted, T2-SPAIR image, and PD-FS.

In the midsagittal plane, the central portion of the plantar plate continued with the intersesamoid ligament on the dorsal aspect of the flexor hallucis longus tendon. We can see a normal hyperintense signal recess at the insertion of the proximal phalangeal base of the central portion of the plantar plate in the midsagittal plane of all five cadaveric feet, which should not be mistaken as a tear (Fig. 2). In the parasagittal plane through the sesamoid bone, the sesamoid phalangeal ligament, metatarsosesamoid ligament, and the flexor hallucis brevis tendon were best demonstrated (Fig. 3). 


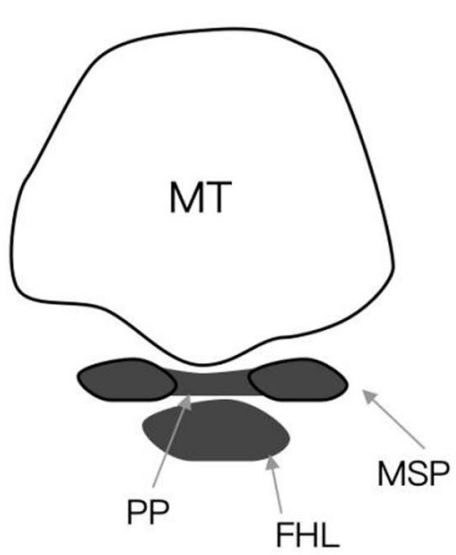

a

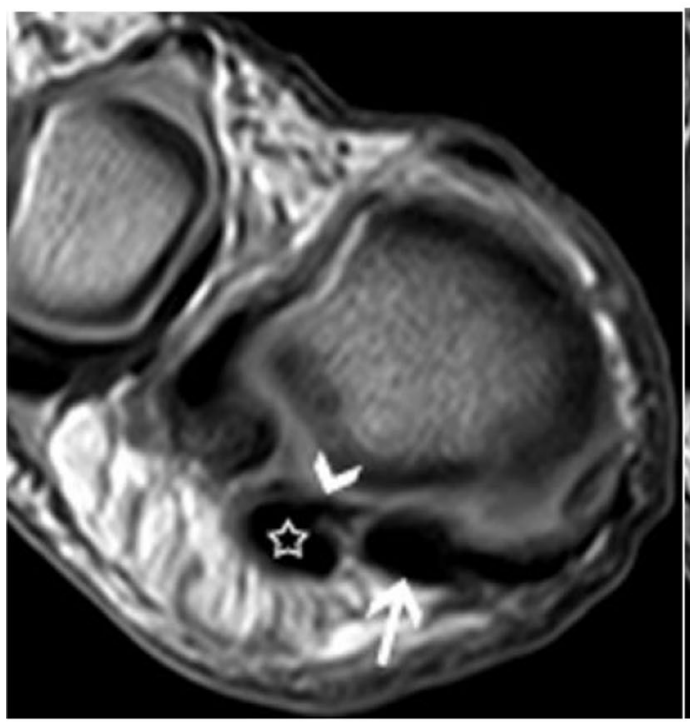

C

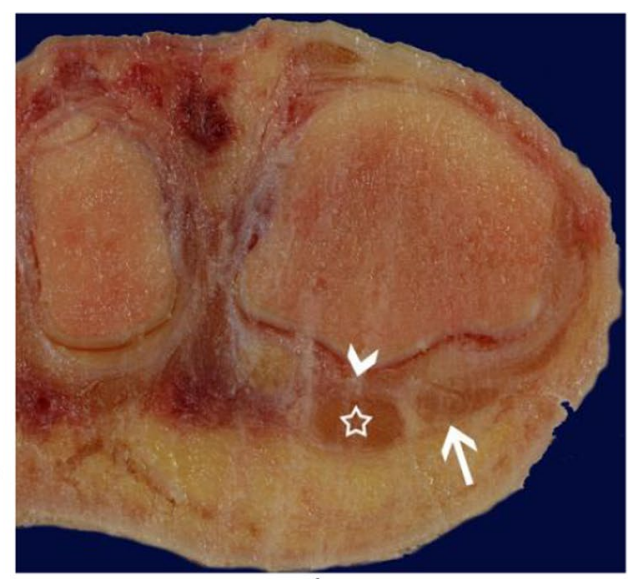

$\mathrm{b}$

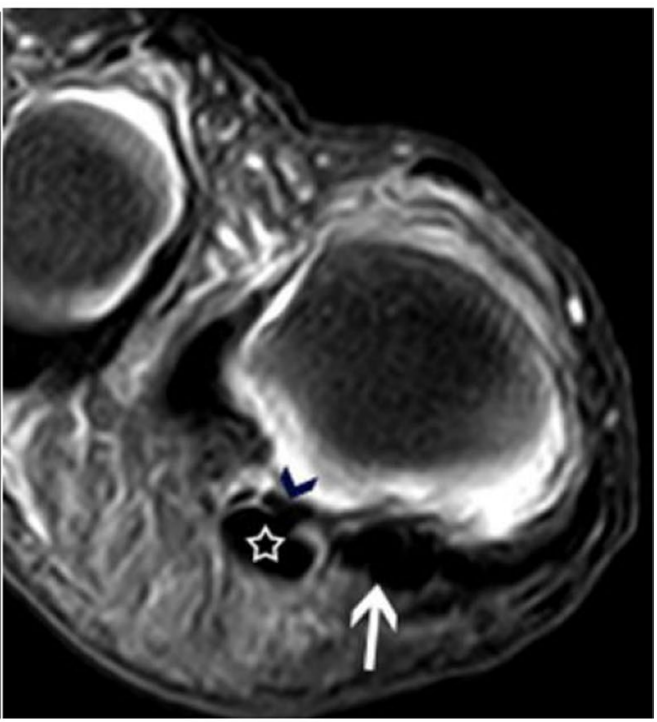

d

Fig. 5 The central portion of the plantar plate and medial sesamoid phalangeal ligament of the first MTPJ from a right foot specimen (45 years old). $\mathbf{a}$ Coronal anatomic comparison slice; $\mathbf{b}$ coronal anatomic slice; $\mathbf{c}$ coronal T1WI image of the foot; $\mathbf{d}$ coronal T2-SPAIR image of the foot. The central portion of the plantar plate (arrowhead) was recognized as curved hypointense signal band on the dorsal of the flexor hallucis longus tendon (white pentagram). (The white arrow = the medial sesamoid phalangeal ligament; $\mathrm{FHL}=$ flexor hallucis longus tendon; MSP $=$ medial sesamoid phalangeal ligament; $\mathrm{MT}=$ metatarsal; $\mathrm{PP}=$ central portion of the plantar plate)

In the coronal plane at the level of the sesamoid bones, the intersesamoid ligament, the accessory sesamoid ligaments, and the supporting structures were all clearly appreciated (Fig. 7). In the coronal plane at the level of the distal to the sesamoid bone, the central portion of the plantar plate manifested as a separate hypointense signal band located between the medial and the lateral sesamoid phalangeal ligaments (Fig. 5).

In the transverse plane at the level of the sesamoid bones, the intersesamoid ligament, the abductor and adductor hallucis tendons were all clearly appreciated (Fig. 8). In the transverse plane at the level of MTPJ, the medial and lateral main collateral ligaments were clearly observed; the lateral one extended distally to the lateral base of the proximal phalanx parallel to the tendon of adductor hallucis (Figs. 6 and 8).

The measurement results of the structures of the capsuloligamentous complex of the first MTPJ are listed in Table 1.

Interobserver agreement ICC values were used to assess interobserver agreement between reader 1 and reader 2 with regard to quantitative measurements. ICC values ranged from 0.46 for the length of the central portion of the plantar plate to 0.97 for the depth of the 


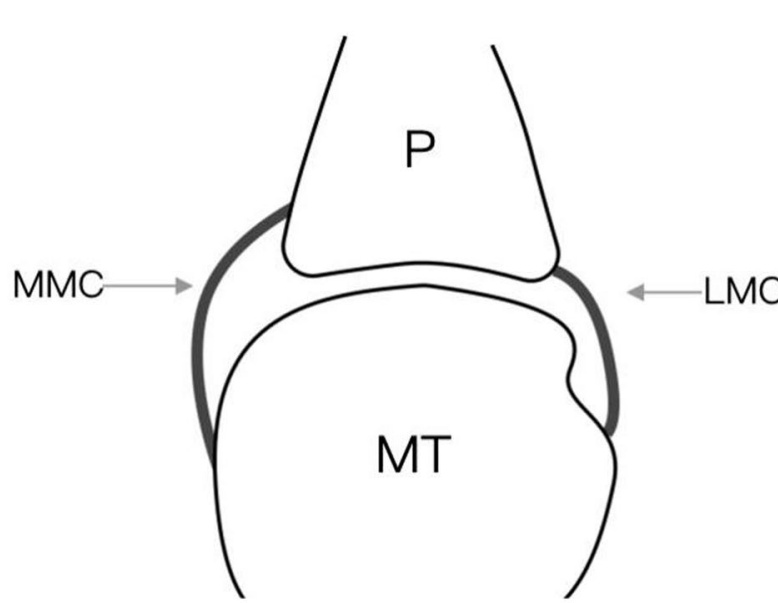

a

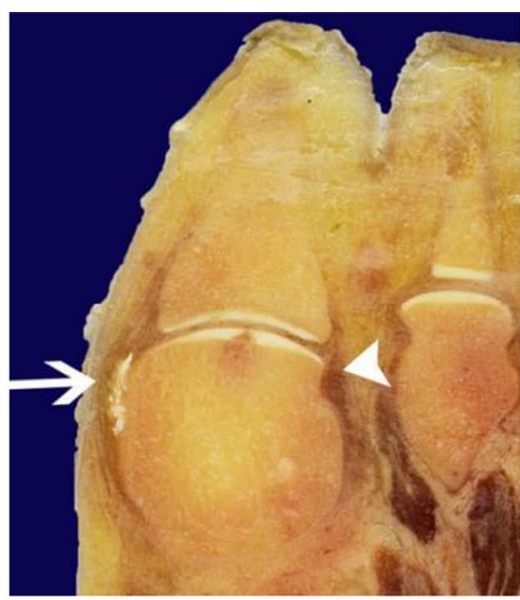

b

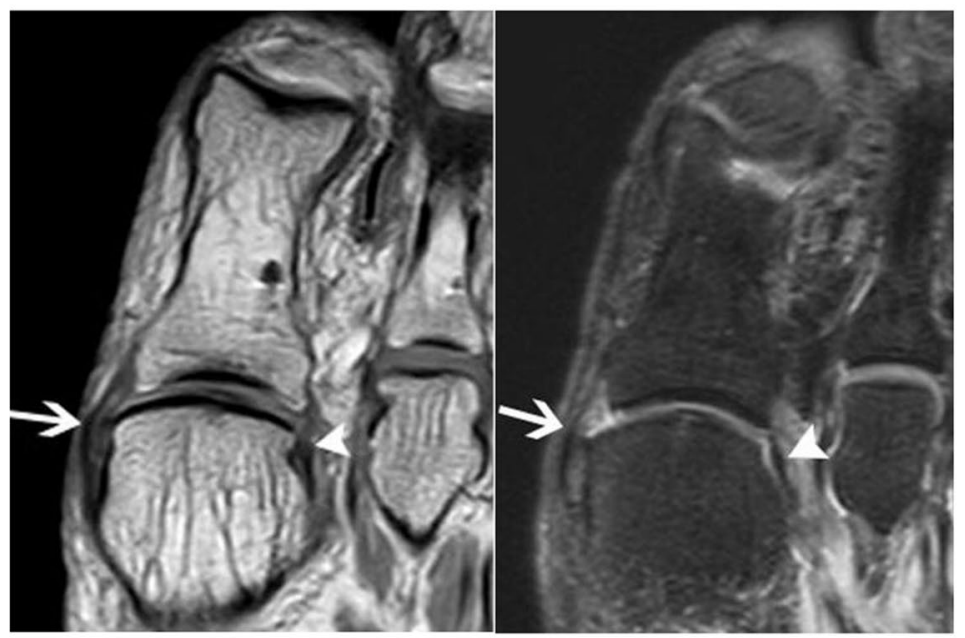

$\mathrm{c}$

d

Fig. 6 The medial and lateral main collateral ligaments from a right foot specimen (33 years old). a Transverse schematic diagram of first MTPJ; $\mathbf{b}$ transverse anatomic slice; $\mathbf{c}$ transverse T1WI image of the foot; $\mathbf{d}$ transverse T2-SPAIR image of the foot. The medial (white arrow) and lateral main collateral ligaments (white arrowhead) were recognized as curved hypointense signal band coursing from the metatarsal head to the proximal phalangeal base. ( $(\mathrm{MC}=$ lateral main collateral ligament; $\mathrm{MMC}=$ medial main collateral ligament; $\mathrm{MT}=$ metatarsal; $\mathrm{P}=$ phalanx.)

\section{(See figure on next page.)}

Fig. 7 The capsuloligamentous complex of the first MTPJ from a right foot specimen (45 years old). a Coronal schematic diagram through the sesamoid bones of first MTPJ; $\mathbf{b}$ coronal anatomic slice; $\mathbf{c}$ and $\mathbf{d}$ coronal T1WI and T2-SPAIR image of the foot; $\mathbf{e}$ and $\mathbf{f} \mathrm{HE}$ and Masson's trichrome staining of the first MTPJ (original magnification $\times 4$ ). White triangle=The intersesamoid ligament; white pentagram = flexor hallucis longus tendon; white arrowheads = the accessory sesamoid ligaments; white short arrows = the medial and lateral head of the flexor hallucis brevis tendons; white long arrow and white curved arrow $=$ The tendons of abductor hallucis and adductor hallucis; white circle $=$ The medial sesamoid phalangeal ligament. (Abd=abductor hallucis tendon; Add=adductor hallucis tendon; FHB-M and FHB-L=medial and lateral head of flexor hallucis brevis tendon; $F H L=$ flexor hallucis longus tendon; $I S$ = intersesamoid ligament; $L=$ lateral sesamoid; $L A S=$ lateral accessory sesamoid ligament; $\mathrm{M}=$ medial sesamoid; $\mathrm{MAS}=$ medial accessory sesamoid ligament; $\mathrm{MT}=$ metatarsal.)

intersesamoid ligament, rated as interobserver agreement from moderate to very good. Most ICC values were rated as good or very good.

\section{Histological study of the capsuloligamentous complex of the first MTPJ}

The histological examination showed that the central 

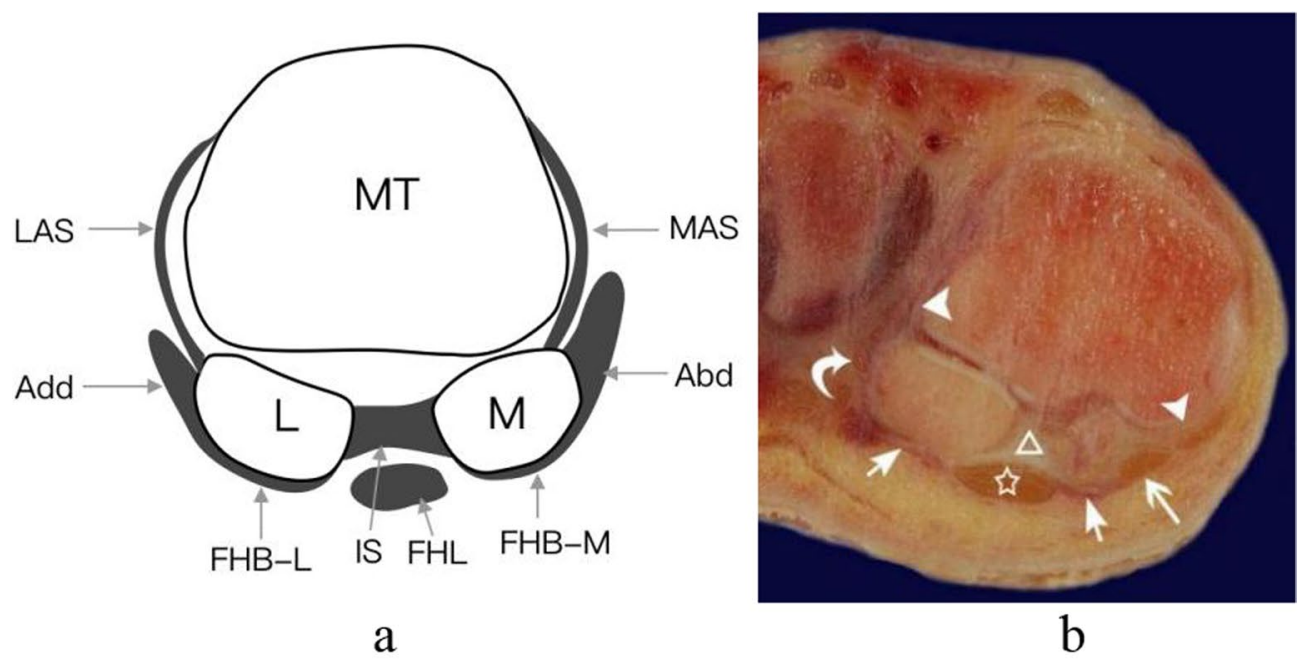

a

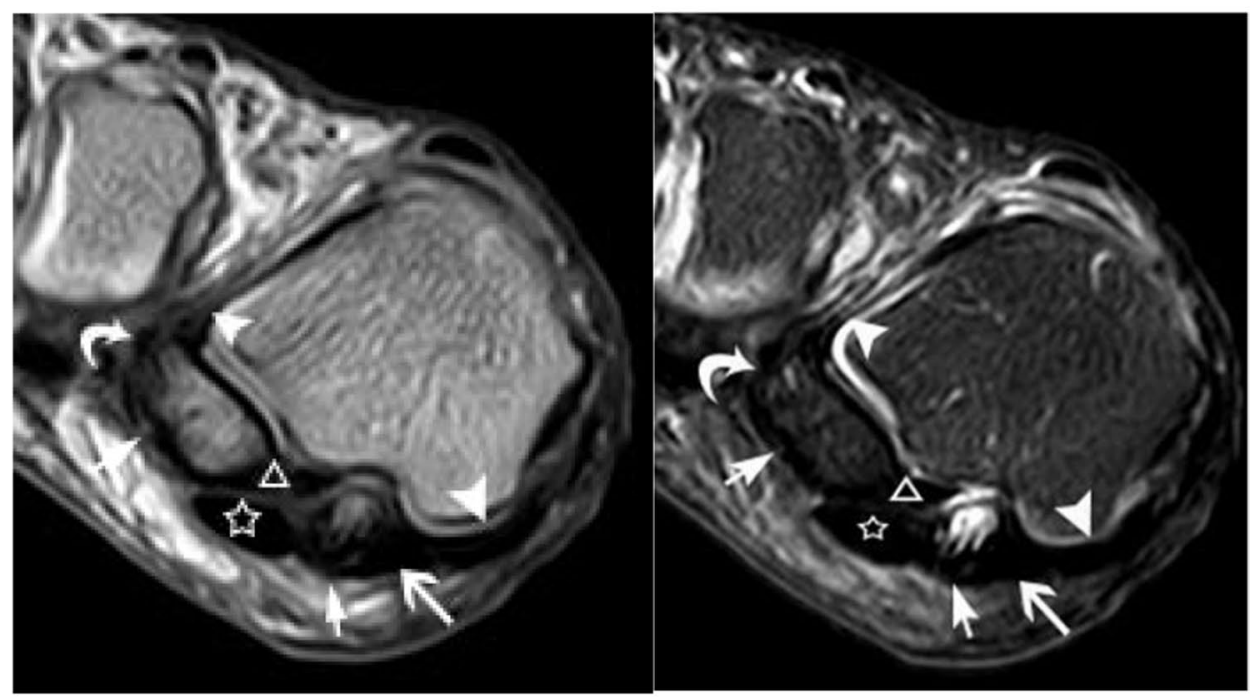

c

d

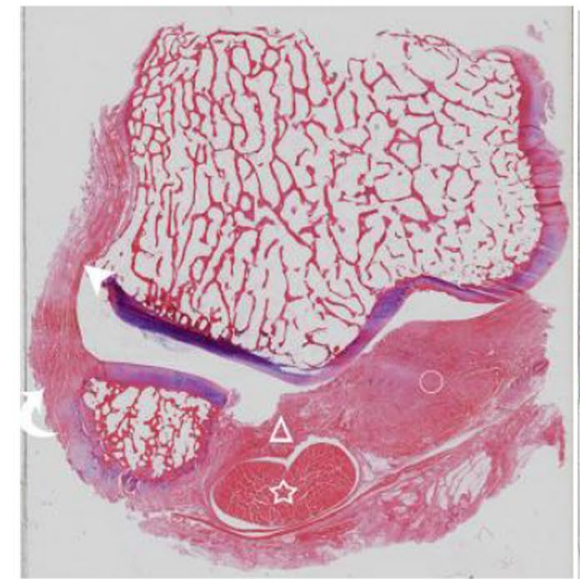

e

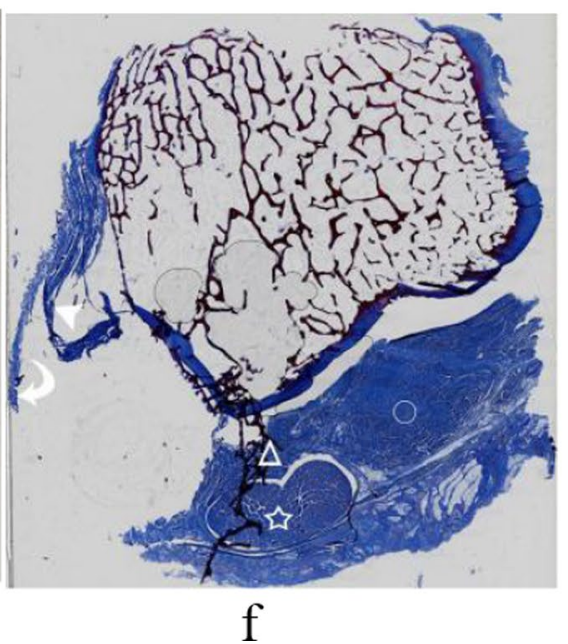

Fig. 7 (See legend on previous page.) 


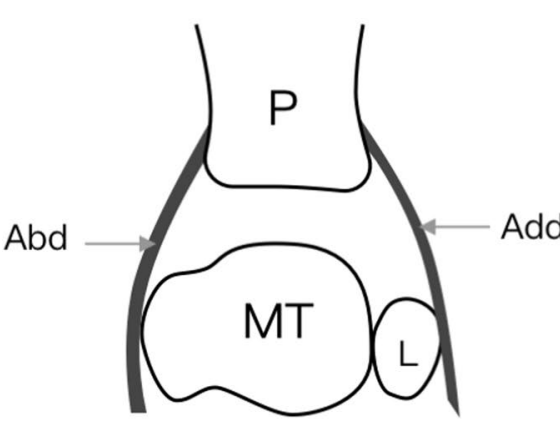

a

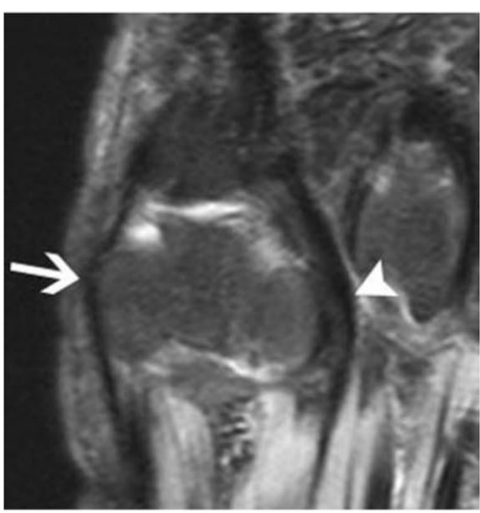

d

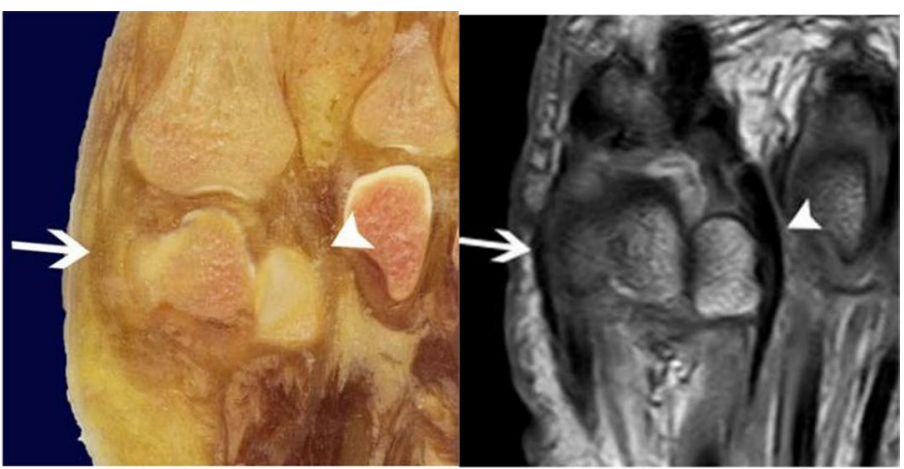

b

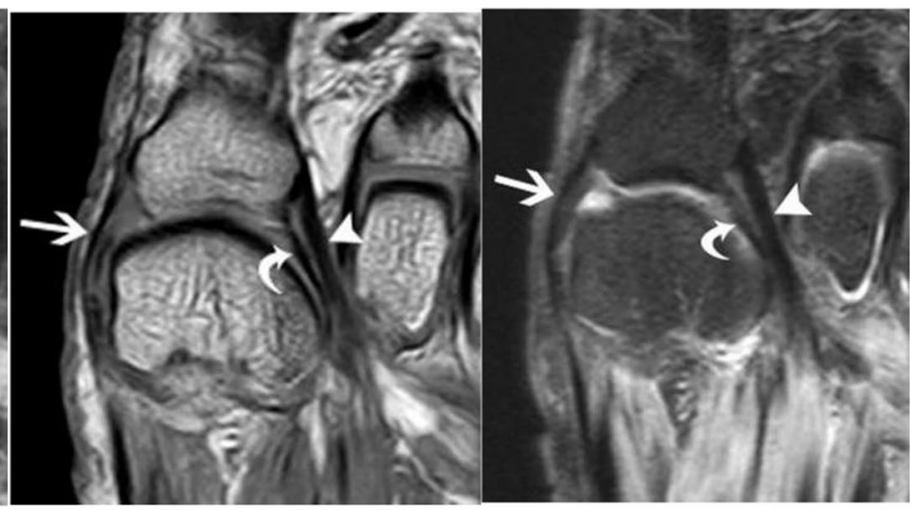

e f

Fig. 8 The tendons of the adductor hallucis and abductor hallucis tendon from a right foot specimen (33 years old). a Transverse schematic diagram of first MTPJ; $\boldsymbol{b}$ transverse anatomic slice; $\mathbf{c}$ and $\mathbf{e}$ transverse T1WI image of the foot; $\mathbf{d}$ and $\mathbf{f}$ transverse T2-SPAIR image of the foot. (White arrow shows the abductor hallucis tendon; white arrowhead shows the adductor hallucis tendon. The lateral main collateral ligament (white curved arrow) extended distally to the lateral base of the proximal phalanx parallel to tendon of adductor hallucis. (Abd=abductor hallucis tendon; Add = adductor hallucis tendon; $\mathrm{L}=$ lateral sesamoid; $\mathrm{MT}=$ metatarsal; $\mathrm{P}=$ phalanx)

portion of the plantar plate, intersesamoid, sesamoid phalangeal and accessory sesamoid ligaments, the tendons of abductor and adductor hallucis of the capsuloligamentous complex of the first MTPJ were all composed of compact collagen fibers. In HE results, the collagen fibers showed eosinophilic features, appeared red in color. The collagen fibers appeared blue with Masson's trichrome staining (Figs. 2, 4 and 7). The central portion of the plantar plate and intersesamoid ligament were composed of the same continuous fiber on the midsagittal slice (Fig. 2e). The central portion of the plantar plate and sesamoid phalangeal ligaments were composed of the same continuous fiber on the coronal slice (Fig. 4). At high magnification $(\times 200)$, the central portion of the plantar plate, intersesamoid and sesamoid phalangeal ligaments appeared interwoven with the same continuous transverse and longitudinal bundles of collagenous fibers with a small number of chondroid metaplasia (Fig. 2). The central portion of the plantar plate, intersesamoid, sesamoid phalangeal and accessory sesamoid ligaments, and the tendons of adductor and abductor hallucis of the capsuloligamentous complex of the first MTPJ showed hypointense signal on all MR images except the normal distal recess of the central portion of the plantar plate that showed hyperintense signal.

\section{Discussion}

The capsuloligamentous complex of the first MTPJ plays an important role in stabilizing the first MTPJ. The complex anatomy of the capsuloligamentous complex of the first MTPJ makes the accurate imaging diagnosis of turf toe very challenge. MRI plays a crucial role in assessing the anatomic details of capsuloligamentous complex of the first MTPJ and has become the most critical imaging modality for visualization of tendons, ligaments, and cartilaginous injuries related to turf toe [1, 2, 10-18]. Our histological analysis of the first MTPJ provides a different understanding of the MRI features from previous studies and clarifies the discrepancy. 
Table 1 Measurement results of the capsuloligamentous complex of the first MTPJ

\begin{tabular}{|c|c|c|c|c|}
\hline & No. & Mean $(\mathrm{mm})$ & $\mathrm{SD}(\mathrm{mm})$ & Range (mm) \\
\hline \multicolumn{5}{|c|}{ Central portion of the plantar plate } \\
\hline Length & 6 & 19.6 & 2.4 & $16.4-22.0$ \\
\hline Width & 7 & 2.9 & 1.3 & $1.4-4.8$ \\
\hline Depth & 8 & 0.5 & 0.2 & $0.3-1.1$ \\
\hline \multicolumn{5}{|c|}{ Intersesamoid ligament } \\
\hline Width & 9 & 3.4 & 0.7 & $2.2-4.2$ \\
\hline Depth & 9 & 2.5 & 0.6 & $1.4-3.4$ \\
\hline \multicolumn{5}{|c|}{ Medial sesamoid phalangeal ligament; } \\
\hline Length & 9 & 11.0 & 2.1 & $7.5-14.3$ \\
\hline Width & 9 & 3.4 & 0.8 & $2.3-4.5$ \\
\hline Depth & 9 & 3.2 & 0.6 & $2.6-4.2$ \\
\hline \multicolumn{5}{|c|}{ Lateral sesamoid phalangeal ligament; } \\
\hline Length & 9 & 7.9 & 0.7 & $6.7-8.8$ \\
\hline Width & 9 & 3.7 & 0.7 & $2.8-5.2$ \\
\hline Depth & 9 & 2.8 & 0.8 & $1.1-3.6$ \\
\hline \multicolumn{5}{|c|}{ Medial metatarsosesamoid ligament; } \\
\hline Length & 5 & 5.4 & 0.9 & $3.9-6.3$ \\
\hline Width & 5 & 0.3 & 0.1 & $0.2-0.4$ \\
\hline \multicolumn{5}{|c|}{ Lateral metatarsosesamoid ligament; } \\
\hline Length & 5 & 6.1 & 1.9 & $3.6-7.9$ \\
\hline Width & 5 & 0.3 & 0.0 & $0.3-0.4$ \\
\hline \multicolumn{5}{|c|}{ Medial main collateral ligament } \\
\hline Length & 9 & 14.5 & 2.0 & $10.1-16.2$ \\
\hline Width & 9 & 2.0 & 0.5 & $1.2-2.7$ \\
\hline \multicolumn{5}{|c|}{ Lateral main collateral ligament } \\
\hline Length & 7 & 12.3 & 2.2 & $9.7-14.7$ \\
\hline Width & 7 & 1.7 & 0.4 & $1.3-2.4$ \\
\hline \multicolumn{5}{|c|}{ Medial head of the flexor hallucis brevis tendon } \\
\hline Width & 3 & 2.5 & 0.7 & $1.9-3.3$ \\
\hline Depth & 3 & 0.4 & 0.2 & $0.3-0.7$ \\
\hline \multicolumn{5}{|c|}{ Lateral head of the flexor hallucis brevis tendon } \\
\hline Width & 2 & 2.9 & 0.4 & $2.6-3.1$ \\
\hline Depth & 2 & 0.3 & 0.1 & $0.3-0.4$ \\
\hline \multicolumn{5}{|c|}{ Adductor hallucis tendon } \\
\hline Width & 5 & 5.7 & 1.6 & $3.4-7.4$ \\
\hline \multicolumn{5}{|c|}{ Abductor hallucis tendon } \\
\hline Width & 5 & 2.8 & 0.3 & $2.4-3.1$ \\
\hline
\end{tabular}

Abbreviations: No, number of the structures; SD, standard deviation

Previous reports $[19,20]$ showed that the plantar plate of the first MTPJ differed from that of the lesser ones $(2-5)$ in that it spanned between the proximal phalangeal base and metatarsal head with the intervening hallux sesamoids. There are discrepancies when describing the components of the plantar plate [11, 13, 16, 21-25]. Some studies $[2,15]$ reported that the plantar plate arose from the distal margin of the intersesamoid ligament and extended distally toward the base of the proximal phalanx. They believed that the plantar plate could not be visualized clearly unless injury caused edema or thickening around it. In our opinion, the plantar plate can be divided into four portions including the central portion of the plantar plate, the intersesamoid, sesamoid phalangeal and metatarsosesamoid ligaments. The central portion of the plantar plate can be clearly visualized in the sagittal and coronal planes in normal cadaver. Other study [1] reported that there was a normal distal recess at the proximal phalangeal insertion of the plantar plate. In our study, we could see the hyperintense signal recess in five cadaveric feet on midsagittal MR images without arthrography, as described in the previous report [11]. This normal appearance should not be recognized as a tear or degeneration. This recess is a smooth, welldefined hyperintense signal region at the proximal phalangeal insertion of the central portion of the plantar plate. The degeneration or tear of the plantar plate usually shows increased signal intensity within the plantar plate, but it is usually poorly defined and irregular.

There are discrepancies when describing the relations between the intersesamoid ligament and the plantar plate in the literature. Allen et al. [6] described that the intersesamoid ligament was on the plantar aspect of the plantar plate. However, the more popular illustration was that the intersesamoid ligament was on the dorsal aspect of the plantar plate [26, 27]. Sanders et al. [28] described that the intersesamoid ligament was the central part of the plantar plate. Tolu et al. [29] illustrated that the intersesamoid ligament was a proximal thickening of the plantar plate. Srinivasan et al. [11] considered that the intersesamoid ligament and the plantar plate were made up of the same contiguous fibrocartilage. In our study, we found that the intersesamoid ligament continued with the central portion of the plantar plate on the sagittal plane of the gross specimen and MR imaging. The central portion of the plantar plate and intersesamoid ligament were composed of the same continuous fiber on the histological slice and appeared interwoven with the same continuous collagenous fibers at high magnification. The previous study reported that the thickness of the intersesamoid ligament was $3.1 \pm 0.5 \mathrm{~mm}$ [16], which was similar to our result $(2.5 \pm 0.6 \mathrm{~mm})$.

In our study, the sesamoid phalangeal ligaments and the central portion of the plantar plate were separate ligaments on the coronal plane of the gross specimen and MR imaging, but they appeared interwoven with the same continuous collagenous fibers on the histological slice. Srinivasan et al. [11] also stated that the sesamoid phalangeal and intersesamoid ligaments were not separate ligaments; they were made up of the same continuous fibrocartilage as the plantar plate. Previous researchers [2] described that sesamoid phalangeal 
ligaments were generally much wider than the metatarsosesamoid ligaments. We found that the length, width, and depth of sesamoid phalangeal ligaments were all greater than those of the metatarsosesamoid ligaments in our study.

Linklater et al. [21] suggested that collateral ligaments were composed of sesamoid collateral ligament and collateral ligament proper. However, Srinivasan et al. [11] had the same view with us that the collateral ligaments consisted of the main collateral ligaments and the accessory sesamoid ligaments. In our study, the mean width of the medial was $(2.0 \pm 0.5 \mathrm{~mm})$, larger than the mean width of the lateral main collateral ligament $(1.7 \pm 0.4 \mathrm{~mm})$. Theumann et al. [1] have the similar result with ours, except that both medial and lateral main collateral ligaments are thicker than ours. In their result, the lateral main collateral ligament $(2.1 \pm 0.2 \mathrm{~mm})$ was thinner than the medial one $(3.1 \pm 0.4 \mathrm{~mm})$. This discrepancy may be due to the ethnic differences of the studied subjects.

So far, few studies have concentrated on the histological feature of the capsuloligamentous complex of the first MTPJ. Brenner et al. [30] illustrated that the intersesamoid ligament was mainly composed of transverse bundles of collagenous fibers with little vertical and longitudinal fiber bundles; the insertion bundles to the sesamoids of the intersesamoid ligament were crisscrossing. Gregg et al. [31] stated that the lesser (2-5) metatarsophalangeal joint plantar plate collagen bundles appeared interwoven with longitudinal fiber bundles. In our study, the central portion of the plantar plate, intersesamoid and sesamoid phalangeal ligaments appeared interwoven with the same continuous transverse and longitudinal bundles of collagenous fibers. Previous reports [32-35] illustrated that normal tendons and ligaments had hypointense signal in all the conventional MRI sequences because the alignment of collagen molecules and water led to dipole interactions that shorten the T2 relaxation time to 1-2 ms and decayed the MR signal. In our study, the central portion of the plantar plate, intersesamoid, sesamoid phalangeal and accessory sesamoid ligaments, and the tendons of adductor and abductor hallucis of the capsuloligamentous complex of the first MTPJ were composed of collagen fibers; they all showed hypointense signal in MRI results except the normal distal recess of the central portion of the plantar plate.

There are some limitations in our study. First, the number of specimens included was relatively small. Second, the capsuloligamentous complex of the first MTPJ of the specimens could be placed in the center of the coil, which was helpful to get better images, but is not always possible in clinical settings. Third, the study did not involve live volunteers, which may provide more anatomical details of the capsuloligamentous complex of the first MTPJ in the real clinical setting.

\section{Conclusions}

In conclusion, using the high-resolution 3T MRI combined with histological analysis, our study clearly illustrated the normal complex anatomy of the capsuloligamentous complex of the first MTPJ, which was different from previous studies. In our study, we found that the central portion of the plantar plate and intersesamoid ligament were composed of the same continuous fiber and the intersesamoid ligament continued with the central portion of the plantar plate and appeared interwoven with the same continuous collagenous fibers. All the structures can be clearly seen on MR images.

\section{Abbreviations}

Abd: Abductor hallucis tendon; Add: Adductor hallucis tendon; Add-O: Oblique head of adductor hallucis tendon; Add-T: Transverse head of adductor hallucis tendon; EDTA: Ethylenediaminetetraacetic acid; FHB: The flexor hallucis brevis; FHB-M: Medial head of flexor hallucis brevis tendon; FHB-L: Lateral head of the flexor hallucis brevis tendon; FHL: Flexor hallucis longus tendon; HE: Hematoxylin and eosin; ICC: Intraclass correlation coefficient; IS: Intersesamoid ligament; L: Lateral sesamoid; LAS: Lateral accessory sesamoid ligament; LMC: Lateral main collateral ligament; LMS: Lateral metatarsosesamoid ligament; LSP: Lateral sesamoid phalangeal ligament; M: Medial sesamoid; MAS: Medial accessory sesamoid ligament; MMC: Medial main collateral ligament; MMS: Medial metatarsosesamoid ligament; MRI: Magnetic resonance imaging; MS: Metatarsosesamoid ligament; MSP: Medial sesamoid phalangeal ligament; MT: Metatarsal; MTPJ: Metatarsophalangeal joint; NBF: Neutral phosphate-buffered formalin; NSA: Number of signals acquired; P: Phalanx; PD-FS: Proton densityweighted imaging with fat suppression; PP: Central portion of the plantar plate; SP: Sesamoid phalangeal ligament; SPAIR: Spectral attenuated inversion recovery; T1WI:T1-weighted image; TE: Echo time; TR: Repetition time.

\section{Supplementary Information}

The online version contains supplementary material available at https://doi. org/10.1186/s13018-021-02795-7.

Additional file 1. Supplementary Table 1. Parameters of standard high resolution 3T MRI Sequences for head-and-neck receiver-only coil (16-channel).

\section{Acknowledgements}

We would like to thank Hu Wei for drawing the computer graphics. All our diagrams were drawn by Wang Jin-e according to the appearance of the corresponding MR images obtained by our institution documented in our PACS. Based on this, these drawings were then computerized by a professional illustrator, Hu Wei.

\section{Authors' contributions}

All authors performed conception and design, or acquisition of data, or analysis and interpretation of data; contributed to drafting the article or reformulating it critically for important intellectual content; done final approval of the version to be published; and agreed to be responsible for all aspects of the work if questions arise related to its accuracy or integrity. JW and ZQ were involved in statistical analysis. Rongjie Bai and Yuming Yin done quality control of data. JW and RB prepared and edited the manuscript. All authors read and approved the final manuscript. 


\section{Funding}

This study was supported by the National Natural Science Foundation of China (Grant numbers 81771809, 82171921), Beijing Natural Science Foundation of China (Grant number 7202063).

\section{Availability of data and materials}

All data generated or analyzed during this study are included in this published article [and its supplementary information files]

\section{Declarations}

\section{Ethics approval and consent to participate}

The study was conducted in accordance with the Declaration of Helsinki and was approved by the Ethics Committee of the Institutional Review Board of Beijing Jishuitan Hospital (201703-10). The authors certify that they have obtained all appropriate consent forms, which were signed by the family members of the donor-cadavers.

\section{Consent for publication}

Not applicable.

\section{Competing interests}

The authors declare that they have no competing interests.

\section{Author details}

${ }^{1}$ Department of Radiology, Peking University Fourth School of Clinical Medicine, Beijing 100035, China. ${ }^{2}$ Department of Radiology, Beijing Jishuitan Hospital, Beijing 100035, China. ${ }^{3}$ Institute of Basic Medical Sciences, Chinese Academy of Medical Sciences, School of Basic Medicine, Peking Union Medical College, Beijing 100005, China. ${ }^{4}$ Direct Radiology, 3501 Ocean Drive, Corpus Christi, TX 78411, USA.

Received: 19 May 2021 Accepted: 12 October 2021

Published online: 24 October 2021

\section{References}

1. Theumann NH, Pfirrmann CW, Mohana Borges AV, Trudell DJ, Resnick D. Metatarsophalangeal joint of the great toe: normal MR, MR arthrographic, and MR bursographic findings in cadavers. J Comput Assist Tomogr. 2002;26:829-38. https://doi.org/10.1097/00004728-20020 9000-00029.

2. Jana MC, Phancao J-P, Stidham K. MR imaging of turf toe. Magn Reson Imaging Clin N Am. 2008;16:93-103. https://doi.org/10.1016/j.mric. 2008.02.002.

3. Lepage-Saucier M, Linda DD, Chang EY, Huang BK, Fliszar EA, Trudell D, et al. MRI of the metatarsophalangeal joints: improved assessment with toe traction and MR arthrography. Am J Roentgenol. 2013;200:868-71. https://doi.org/10.2214/AJR.12.9164.

4. Midfoot GT, Injuries F. Top Magn Reson Imaging. 2015;24:215-21. https://doi.org/10.1097/RMR.0000000000000058.

5. Coughlin MJ, Kemp TJ, Hirose CB. Turf toe: soft tissue and osteocartilaginous injury to the first metatarsophalangeal joint. Phys Sportsmed. 2010:38:91-100. https://doi.org/10.3810/psm.2010.04.1766.

6. Allen LR, Flemming D, Sanders TG. Turf toe: ligamentous injury of the first metatarsophalangeal joint. Mil Med 2004;169:xix-xxiv. PMID: 15605946

7. Clanton TO, Ford JJ. Turf toe injury. Clin Sports Med. 1994;13:731-41 (PMID:7805103)

8. McCormick JJ, Anderson RB. The great toe: failed turf toe, chronic turf toe, and complicated sesamoid injuries. Foot Ankle Clin. 2009;14:13550. https://doi.org/10.1016/j.fcl.2009.01.001

9. McCormick JJ, Anderson RB. Rehabilitation following turf toe injury and plantar plate repair. Clin Sports Med. 2010;29:313-23. https://doi.org/ 10.1016/j.csm.2009.12.010.

10. De Maeseneer M, Moyson N, Lenchik L, Cattrysse E, Scafoglieri A, Roose $\mathrm{R}$, et al. MR imaging-anatomical correlation of the metatarsophalangeal joint of the hallux: ligaments, tendons, and muscles. Eur J Radiol. 2018;106:14-9. https://doi.org/10.1016/j.ejrad.2018.07.003.
11. Srinivasan R. The hallucal-sesamoid complex: normal anatomy, imaging, and pathology. Semin Musculoskelet Radiol. 2016;20:224-32. https://doi.org/10.1055/s-0036-1581121.

12. Waldrop NE, Zirkel CA, Wijdicks CA, Laprade RF, Clanton TO. Radiographic evaluation of plantar plate injury: an in vitro biomechanical study. Foot Ankle Int. 2013;34:403-8. https://doi.org/10.1177/10711 00712464953.

13. Crain JM, Phancao JP. Imaging of Turf Toe. Radiol Clin North Am. 2016:54:969-78. https://doi.org/10.1016/j.rcl.2016.04.010.

14. Wilson L, Dimeff R, Miniaci A, Sundaram M. Radiologic case study. First metarsophalangeal plantar plate injury (turf toe). Orthopedics. 2005;28(344):417-9. https://doi.org/10.3928/0147-7447-20050401-01.

15. Nery C, Baumfeld D, Umans H, Yamada AF. MR Imaging of the plantar plate: normal anatomy, turf toe, and other injuries. Magn Reson Imaging Clin N Am. 2017;25:127-44. https://doi.org/10.1016/j.mric.2016.08. 007.

16. Dietrich TJ, da Silva FLF, de Abreu MR, Klammer G, Pfirrmann CWA. First metatarsophalangeal joint- MRI findings in asymptomatic volunteers. Eur Radiol. 2015;25:970-9. https://doi.org/10.1007/s00330-014-3489-y.

17. Xian JF, Chen M, Jin ZY. Magnetic resonance imaging in clinical medicine: current status and potential future developments in China. Chin Med J. 2015;128:569-70. https://doi.org/10.4103/0366-6999.151637.

18. Bai RJ, Zhang HB, Zhan HL, Qian ZH, Wang NL, Liu Y, et al. Sports injury-related fingers and thumb deformity due to tendon or ligament rupture. Chin Med J. 2018;131:1051-8. https://doi.org/10.4103/03666999.230721.

19. Hockenbury RT. Forefoot problems in athletes. Med Sci Sports Exerc. 1999;31(7 Suppl):S448-458. https://doi.org/10.1097/00005768-19990 7001-00006.

20. Yao L, Do HM, Cracchiolo A, Farahani K. Plantar plate of the foot: findings on conventional arthrography and MR imaging. AJR Am J Roentgenol. 1994;163:641-4. https://doi.org/10.2214/ajr.163.3.8079860.

21. Linklater JM, Hayter CL, Vu D. Imaging of acute capsuloligamentous sports injuries in the ankle and foot: sports imaging series. Radiology. 2017;283:644-62. https://doi.org/10.1148/radiol.2017152442.

22. Burge AJ, Gold SL, Potter HG. Imaging of sports-related midfoot and forefoot injuries. Sports Health. 2012;4:518-34. https://doi.org/10.1177/ 1941738112459489.

23. Drakos MC, Fiore R, Murphy C, DiGiovanni CW. Plantar-plate disruptions: "the severe turf-toe injury." three cases in contact athletes. J Athl Train. 2015;50:553-60. https://doi.org/10.4085/1062-6050-49.6.05.

24. Lucas DE, Philbin T, Hatic S 2nd. The plantar plate of the first metatarsophalangeal joint: an anatomical study. Foot Ankle Spec. 2014;7:10812. https://doi.org/10.1177/1938640014522095.

25. Smith K, Waldrop N. Operative outcomes of grade 3 turf toe injuries in competitive football players. Foot Ankle Int. 2018:39:1076-81. https:// doi.org/10.1177/1071100718775967.

26. David RD, Delagoutte JP, Renard MM. Anatomical study of the sesamoid bones of the first metatarsal. J Am Podiatr Med Assoc. 1989;79:536-44. https://doi.org/10.7547/87507315-79-11-536.

27. Munuera PV, Dominguez G, Polo J, Rebollo J. Medial deviation of the first metatarsal in incipient hallux valgus deformity. Foot Ankle Int. 2006:27:1030-5. https://doi.org/10.1177/107110070602701206.

28. Sanders TG, Rathur SK. Imaging of painful conditions of the hallucal sesamoid complex and plantar capsular structures of the first metatarsophalangeal joint. Radiol Clin North Am. 2008;46:1079-92. https://doi. org/10.1016/j.rcl.2008.09.001.

29. Ashimolowo T, Dunham G, Sharp JW, Porrino J. Turf toe: an update and comprehensive review. Radiol Clin North Am. 2018;56(6):847-58. https://doi.org/10.1016/j.rcl.2018.06.002.

30. Brenner $\mathrm{E}, \mathrm{Gruber} \mathrm{H}$, Fritsch H. Fetal development of the first metatarsophalangeal joint complex with special reference to the intersesamoidal ridge. Ann Anat. 2002;184:481-7. https://doi.org/10.1016/S09409602(02)80082-6.

31. Gregg J, Marks P, Silberstein M, Schneider T, Kerr J. Histologic anatomy of the lesser metatarsophalangeal joint plantar plate. Surg Radiol Anat. 2007;29:141-7. https://doi.org/10.1007/s00276-007-0188-2.

32. Lee HD, Shin JG, Hyun H, Yu BA, Eom TJ. Label-free photoacoustic microscopy for in-vivo tendon imaging using a fiber-based pulse laser. Sci Rep. 2018;8:4805. https://doi.org/10.1038/s41598-018-23113-y. 
33. Chang EY, Szeverenyi NM, Statum S, Chung CB. Rotator cuff tendon ultrastructure assessment with reduced-orientation dipolar anisotropy fiber imaging. AJR Am J Roentgenol. 2014;202:W376-378. https://doi. org/10.2214/AJR.13.11302.

34. Erickson SJ, Cox IH, Hyde JS, Carrera GF, Strandt JA, Estkowski LD. Effect of tendon orientation on MR imaging signal intensity: a manifestation of the "magic angle" phenomenon. Radiology. 1991;181:389-92. https://doi.org/10.1148/radiology.181.2.1924777.
35. Oatridge A, Herlihy AH, Thomas RW, Wallace AL, Curati WL, Hajnal JV, et al. Magnetic resonance: magic angle imaging of the Achilles tendon. Lancet 2001:358:1610-1601. https://doi.org/10.1016/S0140-6736(01)06661-2.

\section{Publisher's Note}

Springer Nature remains neutral with regard to jurisdictional claims in published maps and institutional affiliations.
Ready to submit your research? Choose BMC and benefit from:

- fast, convenient online submission

- thorough peer review by experienced researchers in your field

- rapid publication on acceptance

- support for research data, including large and complex data types

- gold Open Access which fosters wider collaboration and increased citations

- maximum visibility for your research: over $100 \mathrm{M}$ website views per year

At BMC, research is always in progress.

Learn more biomedcentral.com/submissions 